Wissenschaftliche Beiträge

\title{
Die Integration materieller und prozessualer Elemente im Verwaltungsrecht
}

\section{Ihre Rechtfertigung und die Alternative eines anspruchsbasierten Formats}

\author{
Andreas Funke"
}

\begin{abstract}
Zusammenfassung: Das Öffentliche Recht nimmt in Lehre und Prüfung gegenüber dem Zivilrecht und dem Strafrecht insofern eine Sonderstellung ein, als es von vorneherein das Prozessrecht einbezieht. Thema sind stets die Erfolgsaussichten eines Rechtsbehelfs. Der Beitrag untersucht, konzentriert auf das Verwaltungsrecht, wie sich diese Praxis entwickelt hat und wie sie zu rechtfertigen ist. Überzengende Gründe finden sich nicht. Im Gegenteil: Wichtige systematische Zusammenhänge werden durch das integrative Format verdunkelt. Der Beitrag schlägt die Alternative eines anspruchsorientierten Formats vor, das die im Verhältnis von Verwaltung und Bürger bestehenden Rechte und Pflichten in den Mittelpunkt rückt.
\end{abstract}

\section{A. Einleitung}

Das Jurastudium ist im Wesentlichen darauf gerichtet, die richtige Lösung von praktischen Fällen zu vermitteln. Dies gilt für alle drei Fachsäulen, also das Zivilrecht, das Strafrecht und das Öffentliche Recht. Der Grund für diese Ausrichtung ist bekanntermaßen darin $\mathrm{zu}$ suchen, dass das Studium nicht (allein) mit einer universitären Prüfung, sondern mit dem Staatsexamen abgeschlossen wird, in dem vor allem Praktiker/-innen und nur ergänzend Hochschullehrer/-innen prüfen. ${ }^{1}$ Erstaunlicherweise unterscheiden sich aber die Fachsäulen in der Rolle, die dem Prozessrecht zukommt. Die Lösung zivilrechtlicher Fälle ist regelmäßig materiellrechtlich, am Bestehen von Ansprüchen, ausgerichtet, die Lösung strafrechtlicher Fälle an der Strafbarkeit einer bzw. mehrerer Personen, verstanden als materiellrechtliche Qualifikation eines bestimmten Geschehens. Im Zivilrecht wie im Strafrecht ist das Prozessrecht allenfalls Gegenstand einer Zusatzfrage.

Anders im Öffentlichen Recht. Sowohl im Verfassungs- als auch im Verwaltungsrecht wird von vorneherein das Prozessrecht in die Lehre wie auch in die Prüfung integriert. Angeblich sind 90\% der Aufgabenstellungen im Öffentlichen Recht auf die Erfolgsaussichten einer verwaltungsgerichtlichen Klage gerichtet. ${ }^{2}$ Vermutlich liegt der Anteil noch höher. Die Ausbildungsliteratur geht auf dieser Grundlage den Weg der Totalintegration, d. h. sämtliche Aufgabenstellungen werden aus der

* Prof. Dr. Andreas Funke, Inhaber des Lehrstuhls für Öffentliches Recht und Rechtsphilosophie, Friedrich-Alexander-Universität Erlangen-Nürnberg.

1 Zur geschichtlichen Entwicklung Rinken, Einführung in das juristische Studium, S. 122 ff.; zur Rolle von Fallübungen Haferkamp, in: ZRGG 138 (2021), S. 283.

2 Eisentraut, in: ders. (Hrsg.), Verwaltungsrecht in der Klausur, S. XXXVII (XXXVII). 
Perspektive der Erfolgsaussichten eines Rechtsbehelfs dargestellt. ${ }^{3}$ Es gilt als didaktische Tugend, den Stoff in diesem integrativen Format zu lehren. ${ }^{4}$ An etwa der Hälfte der Jurafakultäten wird das Allgemeine Verwaltungsrecht gemeinsam mit dem Verwaltungsprozessrecht in einer Vorlesung behandelt. ${ }^{5}$ Studierende verinnerlichen schnell, dass es im Öffentlichen Recht um „Zulässigkeit und Begründetheit“ geht. Wer als Hochschullehrerin darauf verzichtet, irritiert, nicht nur Studierende, sondern auch Tutor/-innen und wissenschaftliche Mitarbeiter/-innen, die mit Arbeitsgemeinschaften bzw. Propädeutischen Übungen betraut sind. Die Situation ist geradezu paradox: Aufgabenstellungen, die auf eine prozessuale Einkleidung verzichten, bereiten den Studierenden häufig mehr Schwierigkeiten. Rufe danach, die Rolle zu überdenken, die das Verwaltungsprozessrecht im Lehr- und Prüfprogramm spielt, ${ }^{6}$ sind ungehört verhallt.

Dass „der bloße Klausurenjurist“, den die juristische Ausbildung produziert, eine problematische Erscheinung ist, ${ }^{7}$ ist hinlänglich bekannt und soll hier nicht erneut diskutiert werden. Aber wenn schon Klausuren, dann die richtigen! Was ist denn eigentlich der Sinn der Verknüpfung von Verwaltungsprozessrecht und materiellem Verwaltungsrecht? Wie ist es zu rechtfertigen, dass das Öffentliche Recht diesen Unterschied zu den anderen Fachsäulen aufweist? Welche spezifische Kompetenz sollen sich die Studierenden damit aneignen?

Diesen Fragen geht der Beitrag nach. Das mag nutzlos erscheinen. Die eingefahrene Praxis wird sich kaum ändern lassen. $\mathrm{Zu}$ stark sind die Gewohnheiten der Prüfer wie auch der Lehrenden. Eine Beschäftigung mit dem Thema läuft Gefahr, nur nachträglich einen Entwicklungspfad zu rationalisieren, der nie bewusst eingeschlagen wurde, aber auch nicht mehr verlassen werden kann. Diese Gefahr realisiert sich in diesem Beitrag allerdings nicht, weil er zu dem Ergebnis gelangt, dass es keine überzeugenden Gründe für die aktuelle Praxis gibt. Ich möchte vielmehr vorschlagen, in der Lehr- und Prüfungspraxis die beiden Ebenen des materiellen Verwaltungsrechts (weit verstanden, so dass das Verwaltungsverfahrensrecht einbezogen ist) und des Verwaltungsprozessrechts deutlicher voneinander zu trennen.

Zwei thematische Einschränkungen seien vorangeschickt: Der Beitrag konzentriert sich erstens auf das Verwaltungsrecht und vernachlässigt das Verfassungsrecht. Die prozessualen Durchsetzungsmöglichkeiten beim BVerfG haben eine institutionelle Dimension, die für das Verständnis des Verfassungsrechts außerordentlich wichtig

3 Siehe etwa Seidel/Reimer/Möstl, Allgemeines Verwaltungsrecht; Uerpmann-Wittzack, Examens-Repetitorium; Heinze, Systematisches Fallrepetitorium; Brüning/Suerbaum, Examensfälle; Wolf, Fälle und Lösungen; Schoch, Übungen II; Brinktrine/Kastner, Fallsammlung; Eisentraut (Hrsg.), Fälle, Dietlein/Burgi/Hellermann, Klausurenbuch; Sauer, Klausurtraining, Rn. 30; Peine, Klausurenkurs, Rn. 23. Breiter hingegen Bretthauer/Collin u. a., 40 Klausuren; Böhm/Gaitanides, Fälle; Winkler, Klausurtraining; Muckel, Fälle; Becker/Heckmann u. a., Klausurenbuch.

4 Englisch/Cryns, Fälle und Lösungen, S. 5.

5 Sauer, in: Krüper (Hrsg.), Rn. 5.

6 Bull, in: JZ 1998, S. 338.

7 Begriff bei Ridder/Schmidt, in: JuS 1966, S. 237 (238). Zum (Gegen-)Leitbild der juristischen Bildung siehe Funke, in: Krüper (Hrsg.), Rn. 22 ff. 
ist. Damit sind Unterschiede zum Verwaltungsrecht verbunden, die eine Sonderstellung des Verfassungsprozessrechts rechtfertigen mögen. Zweitens lassen die Überlegungen den Stoff, der zum Verwaltungsrechtscurriculum zählt, unangetastet. ${ }^{8} \mathrm{Zu}$ den Vorschlägen, daran etwas zu ändern, etwa mit Blick auf eine stärkere Einbeziehung sozialwissenschaftlicher Perspektiven, ${ }^{9}$ verhalten sie sich neutral.

\section{B. Rückblick}

\section{Die Tradition der Integration: eine kursorische Umschau}

Es ist naheliegend, in einem ersten Zugang zum Thema rückblickend aufzuarbeiten, wie es überhaupt zur Verknüpfung von Verwaltungsprozessrecht und materiellen Verwaltungsrecht gekommen ist. Das ist jedoch alles andere als einfach.

Die einschlägigen rechtlichen Regelungen der Juristenausbildung sind nicht ergiebig. Soweit ersichtlich, wurde bislang weder in Juristenausbildungsordnungen noch in universitären Studien- und Prüfungsordnungen geregelt, welche Gestalt die Prüfungsaufgaben haben. Es handelt sich um eine Angelegenheit der universitären Praxis, die in Wechselwirkung mit der Prüfungspraxis steht. Die rechtlichen Regelungen gaben stets nur die üblichen Leitbilder der Ausbildung vor und definierten den Prüfungsstoff. Nicht aber legten sie fest, in welcher Form der Stoff geprüft werden soll. Der einzige, aber nicht bedeutsame normative Anknüpfungspunkt ist die Aufnahme von Übungen in die universitäre Lehre, die sich gegen Ende des 19. Jahrhunderts auf der Grundlage von Änderungen der Studienordnungen vollzog. Sie beförderte offenbar die Fallorientierung des Studiums, einschließlich der entsprechenden Prüfungen. Aber damit war nicht die Vorgabe verbunden, in den Übungen im Öffentlichen Recht, anders als in denjenigen im Zivilrecht und im Strafrecht, das Prozessrecht zu integrieren.

Dokumentationen der universitären Lehrpraxis im 19. und 20. Jahrhundert sind für den hier interessierenden Bereich kaum vorhanden. Lehrbücher stellen die systematische Ordnung des Stoffs in den Vordergrund und helfen deshalb nicht weiter. Was bleibt, ist eine Sichtung solcher Lehrmaterialien, die für die universitäre Lehre sowie die entsprechenden Prüfungen verfasst wurden. Eine Reflexion der Art der Aufgabenstellung findet darin zwar kaum statt, aber auf der Grundlage gelegentlicher Bemerkungen und der konkret gewählten Aufgabenstellung lässt sich ein Bild zusammensetzen. Es ist allerdings recht bunt.

Nur für das Zweite Staatsexamen schien relativ früh klar zu sein, dass die Prüfung mit der Einbeziehung des Öffentlichen Rechts - und damit v. a. des Verwaltungsrechts - an der gerichtlichen Entscheidung auszurichten war. ${ }^{10} \mathrm{Im}$ Zivilrecht und

8 Dazu Franzius, in: ZDRW 2 (2015), S. 93; Sauer, in: Krüper (Hrsg.).

9 Franzius, in: ZDRW 2 (2015), S. 93; fallorientiert Hoffmann-Riem (Hrsg.), Sozialwissenschaften im öffentlichen Recht; Lomfeld (Hrsg.), Die Fälle der Gesellschaft (Fälle 4-6).

10 Schrödter, Die verwaltungsrechtliche Entscheidung; Klein, Gutachten und Urteil. Anders, d. h. nur mit punktueller Einbeziehung des Prozessrechts, das offenbar für Referendare angefertigte Werk Kollath, Praktische Verwaltungsrechtsfälle. 
im Strafrecht war das so, das Öffentliche Recht zog nur gleich. Für das Erste juristische Staatsexamen war hingegen im Öffentlichen Recht schon die Fallorientierung als solche nicht von Anfang an selbstverständlich. Neben einer fallorientierten Aufgabe stand in manchen Ländern (Bayern, Baden-Württemberg) eine Themenklausur. ${ }^{11}$ Man kann dem heute nur noch nostalgisch nachtrauern. „Warum jetzt Verfassung? “, auf diese wunderbare Frage mussten beispielsweise die Examenskandidaten (und vermutlich auch -kandidatinnen) im Jahr 1947 in Bayern antworten. ${ }^{12}$ Im Laufe der 1960er Jahre ging die Bedeutung der Themenklausur zurück. ${ }^{13}$

Für die fallorientierte Aufgabe war selbst in den 1970er Jahren das heute selbstverständliche integrative Format noch nicht durchgehend vertreten. Stern konzipiert zwar schon 1965 den „Verwaltungsrechtsfall“ umstandslos so, dass anhand des Schemas von Zulässigkeit und Begründetheit die Erfolgsaussichten eines Rechtsbehelfs zu prüfen sind. ${ }^{14}$ Auch Schwerdtfeger orientiert sich 1969 von vorneherein am, wie er es nennt, Prozessschema, und meint, dass zwar nicht alle, aber viele öffentlich-rechtliche Klausuren daran ausgerichtet sind. ${ }^{15}$ Achterberg wählt 1975 ohne Weiteres das integrative Format. ${ }^{16}$

Andere Lehrwerke weichen davon ab. Sie enthalten eine Mixtur von Aufgabenstellungen. Zum Teil kann dabei ein Muster identifiziert werden, bei dem drei Arten von Fragen zumindest den Kern bilden: die Erfolgsaussichten von Rechtsbehelfen (Zulässigkeit und Begründetheit), das Bestehen von Schadenersatz- und Entschädigungsansprüchen (evtl. ergänzt um vertragliche Ansprüche) und die Rechtmäßigkeit ergangener behördlicher Entscheidungen. ${ }^{17}$ Zum Teil kommt noch die Frage danach hinzu, wie die Behörde in Anbetracht einer bestimmten Sachlage entscheiden soll. ${ }^{18}$ Die übrigen Lehrwerke enthalten ganz diverse Aufgabenstellungen: Erfolgsaussichten eines Rechtsbehelfs (wobei die Zulässigkeitsprüfung häufig wegfällt oder kursorisch behandelt wird), prozessuale oder materielle Einzelfragen,

11 Siehe die Beispiele für Baden-Württemberg in Kern/Widenmann, Das juristische Studium in BadenWürttemberg, S. 55 ff.; für Bayern eine Aufzählung der Themenaufgaben der Jahre 1947-1964 in: JuS 1964, S. 414 (414-416); der Jahre 1964-1966 in: JuS 1967, S. 238 (238). Im Nationalsozialismus dominierte - im Rahmen der reichseinheitlichen Juristenausbildung - sogar die Themenaufgabe, siehe Würfel, Das Reichsjustizprüfungsamt, S. 155. „Klassisch“ fallorientiert hingegen die Ausrichtung bei Redelberger, Das öffentliche Recht (mit groteskem Inhalt); Schneider, Öffentliches Recht (mit einer Liste von Themenaufgaben).

12 JuS 1964, S. 414 (414).

13 Sie gelten als selten bei Ridder/Schmidt, in: JuS 1966, S. 237 (237).

14 Stern, in: JuS 1965, S. 139 (139).

15 Schwerdtfeger, in: JuS 1969, S. 472 (473).

16 Achterberg, Fälle und Lösungen nach höchstrichterlichen Entscheidungen. Freilich ist die Darstellung gerade an Entscheidungen ausgerichtet.

17 So bei Vogel, Der Verwaltungsrechtsfall (1960) (fortgeführt bis zur neubearbeiteten 8. Aufl. 1980, die zwar teilweise andere Fälle enthält, aber die Form der Aufgabenstellungen weitgehend beibehalten hat); von Münch, Übungsfälle; Hans, Öffentliches Recht II (1975); Schmidt-Jortzig/Ipsen, 30 Klausuren; Erbel, Öffentlich-rechtliche Klausurenlehre, Bd. 2.

18 So Fleiner, Verwaltungsrechtsfälle zum akademischen Gebrauch; Vogel, Die verwaltungsrechtliche Hausarbeit (die beiden Aufgaben nehmen die behördliche Entscheidungsperspektive ein). 
Rechtmäßigkeit. ${ }^{19}$ Zum Teil werden ausdrücklich prozessuale Fragen gestellt, neben materiell-rechtlichen. Ähnlich ist das Bild, das eine Dokumentation der Lage in Baden-Württemberg in den Jahren 1953 bis 1957 vermittelt. $^{20}$

Auffallend ist, dass für die Einbeziehung des Verwaltungsprozessrechts zum Teil anstelle der Integration ein anderes Format gewählt wurde. Man kann es akzessorische Stufung nennen. Es behandelt die prozessuale Seite in strikter Akzessorietät zur materiellen Rechtslage. Zunächst ist die materiell-rechtliche Rechtslage zu würdigen, sodann sind, abhängig vom Ergebnis der materiell-rechtlichen Seite, die möglichen Rechtsbehelfe zu erläutern. ${ }^{21}$

Der Schwierigkeitsgrad prozessualer Aufgabenstellungen wurde unterschiedlich beurteilt. Von Münch hält sie im Jahr 1967 für anspruchsvoll; das Verwaltungsprozessrecht werde von Studierenden mangels praktischer Erfahrung oft nur schwer verstanden. ${ }^{22}$ In den Anfängerübungen würden häufig allein materiell-rechtliche Fragen behandelt, während in den Fortgeschrittenenübungen oft das Prozessrecht hinzukomme. ${ }^{23}$ Stern sieht im Prozessrecht hingegen etwas, das der Student erarbeiten und beherrschen kann, im Unterschied zum unvorhersehbaren materiellen Teil. ${ }^{24}$

\section{Die Entwicklung im Kontext von verwaltungsrechtlicher Dogmatisierung und Rechtsschutzgarantie}

Die vorstehenden Beobachtungen zeigen, dass eine Entwicklung hin zur mehr oder weniger vollen Integration des Prozessrechts stattgefunden hat. Verschiedene Faktoren dürften dabei wirksam geworden sein.

Nicht ohne Weiteres lässt sich ein Bezug dazu herstellen, wie sich die Art und Weise der wissenschaftlichen Durchdringung des Verwaltungsrechts seit dem letzten Drittel des 19. Jahrhunderts entwickelt hat. Die zu jener Zeit einsetzende Dogmatisierung des Verwaltungsrechts ist nicht unmittelbar mit der Entstehung und Verbreitung des verwaltungsgerichtlichen Rechtsschutzes verbunden. Beide Entwicklungen verliefen vergleichsweise unabhängig voneinander. Beispielsweise erarbeitete Otto Mayer, dessen Werk für die Etablierung der dogmatischen Methode im Verwaltungsrecht steht, seine Darstellung weitgehend unabhängig vom Verwaltungsprozessrecht. Der verwaltungsgerichtliche Rechtsschutz war für ihn

19 Ksoll, Verwaltungsrechtsfälle und Klausuren; Hans, Öffentliches Recht II (1960); Weimar, Staatsund Verwaltungsrecht (für Verwaltungs- und Wirtschaftsakademien).

20 Kern/Widenmann, Das juristische Studium in Baden-Württemberg.

21 Vgl. von Münch, Übungsfälle, S. 25; Ule, in: JuS 1961, S. 190 (193, 194); Ridder/Schmidt, in: JuS 1966, S. 237 (240); Schneider, in: VDStRL (Hrsg.), S. 28 (31). Beispiele bei Vogel, Der Verwaltungsrechtsfall (1960/1980), Fall 7; Schmidt-Jortzig/Ipsen, 30 Klausuren, verschiedene Fälle.

22 von Münch, Übungsfälle, S. 147.

23 von Münch, Übungsfälle, S. $24 \mathrm{f}$.

24 Stern, in: JuS 1965, S. 139 (139). 
eines der Institute des Verwaltungsrechts, ohne systembildend zu wirken. ${ }^{25}$ Für den Begriff des Verwaltungsakts spielte die Art des mit dem Verwaltungsakt verknüpften Rechtsschutzes bei Mayer keine Rolle. Zugleich ist aber unübersehbar, dass sich Ende des 19. Jahrhunderts das Interesse an der dogmatischen Durchdringung des Verwaltungsrechts auch aus dem Bedürfnis speiste, der Durchsetzung des Verwaltungsrechts in der Form von subjektiven Rechten gerecht zu werden. In Georg Jellineks „System der subjektiven öffentlichen Rechte“, in der ersten Auflage 1892 erschienen, wirkt die Etablierung des verwaltungsgerichtlichen Rechtsschutzes als ein zentrales Motiv für die Systembildung. ${ }^{26}$ Die Verwissenschaftlichung des Verwaltungsrechts verlief also durchaus im Wechselspiel mit dem Entstehen einer unabhängigen Verwaltungsgerichtsbarkeit. Aber wie genau sich dies in der universitären Lehre und im Prüfungswesen niederschlug, lässt sich nur schwer rekonstruieren.

In der Zeit nach dem Inkrafttreten des Grundgesetzes gewann die prozessuale Perspektive überragende Bedeutung. „Umfassenden Schutz gegen rechtswidrige Eingriffe der öffentlichen Gewalt zu gewähren, war die Devise. “,27 so konstatiert Stolleis für das erste Jahrzehnt der Bundesrepublik. Das Verwaltungsrecht wurde "gerichtsorientiert". ${ }^{28}$ Mit der Aufnahme der bedingungslosen Garantie des Rechtswegs nach Art. 19 Abs. 4 GG bestand nach allgemeiner Auffassung eine auch gegenüber der Weimarer Republik neue Lage, die rechtspraktisch wie rechtswissenschaftlich zu bewältigen war. Die Verwaltungsgerichtsbarkeit wuchs, die begleitende Literatur explodierte. Die Gravitationskraft des Art. 19 Abs. 4 GG reichte vermutlich bis in die Prüfungsämter und in die juristischen Fakultäten, die über die Gestalt verwaltungsrechtlicher Aufgabenstellungen zu entscheiden hatten.

Aber die verfassungsrechtliche Rechtsschutzgarantie spielte - und spielt - insofern eine eigenartige Rolle. Gunther Schwerdtfeger sah im Jahr 1969 die Besonderheit des Öffentlichen Rechts darin, dass dort die gerichtliche Rechtsdurchsetzung, anders als im Zivilrecht, nicht selbstverständlich sei. ${ }^{29}$ Diese Einschätzung findet sich noch heute in seinem Studienbuch, auch in der Neubearbeitung durch Angela Schwerdtfeger: Es soll im Öffentlichen Recht zweifelhaft sein können, „ob wegen der Systementscheidung für subjektiven Rechtsschutz (s. Art. 19 IV 1 GG) eine Klagemöglichkeit besteht ". ${ }^{30}$ Die Aussage enthält einen offensichtlichen Widerspruch: Weil Art. 19 Abs. 4 GG den Rechtsschutz garantiert, sofern ein subjektives öffentliches Recht besteht, ist doch ohne Zweifel eine Klagemöglichkeit gegeben. Mangels Einschlägigkeit des Art. 19 Abs. 4 GG müsste dann eher im Zivilrecht die Selbstverständlichkeit der gerichtlichen Durchsetzung bröckeln. Aber für zivil-

25 Mayer, Deutsches Verwaltungsrecht, Bd. 1, S. $122 \mathrm{ff}$.

26 Vgl. die Bemerkung in Jellinek, System, S. 4 f. Näher Funke, in: Brocker (Hrsg.).

27 Stolleis, in: GVwR I, $\mathbb{} 2$ Rn. 106.

28 Wahl, Herausforderungen und Antworten, S. 39.

29 Schwerdtfeger, in: JuS 1969, S. 472 (473).

30 Schwerdtfeger/Schwerdtfeger, Öffentliches Recht in der Fallbearbeitung, $\mathbb{} 1$ Rn. 4. 
rechtliche Ansprüche greift der Justizgewährungsanspruch, ${ }^{31}$ so dass im Ergebnis kein Unterschied zum Öffentlichen Recht besteht.

Vermutlich zielt der Hinweis auf Art. 19 Abs. 4 GG auf etwas anderes. Hinter dem integrativen Format steht die Annahme, erst die gerichtliche Rechtsdurchsetzung würde den Studierenden das Panorama des Verwaltungsrechts vor Augen führen. ${ }^{32}$ Die Studierenden müssten wissen, wie die subjektiven öffentlichen Rechte gegen den Staat durchgesetzt werden. Aber dann stellt sich erneut die Frage, wo insofern der Unterschied zum Zivilrecht und zum Strafrecht liegt. In der Rechtspraxis kommt es in diesen Bereichen nicht mehr und nicht weniger auf das Prozessrecht an. Und selbst wenn man davon ausgeht, dass die verwaltungsgerichtliche Rechtsdurchsetzung unverzichtbar ist: Es könnten dann immer noch das materielle Recht und das Prozessrecht nebeneinander - oder wie in der „akzessorischen Stufung“: hintereinander - vermittelt werden. So geschieht es nun einmal im Zivilrecht und im Strafrecht. Gibt es andere Gründe für die Sonderstellung des Verwaltungsrechts?

\section{Die Verzahnungsthese}

Das wohl zentrale Argument für die Wahl des integrativen Formats lautet, dass das Verwaltungsprozessrecht und das materielle Verwaltungsrecht miteinander verzahnt sind. Diese Verzahnungsthese hat bei genauerem Hinsehen zwei verschiedene Bedeutungen: Nach einer starken Fassung sind bestimmte Elemente innerlich miteinander verknüpft. Nach der schwachen Fassung fehlt es zwar an einer solchen Verknüpfung, aber die eine Rechtsmaterie kann besser verstanden werden, wenn die jeweils andere Rechtsmaterie in den Blick genommen wird.

\section{Die schwache Fassung}

Die schwache Fassung der These der Verzahnung von materiellem Verwaltungsrecht und Prozessrecht kann in beide Richtungen weisen. So wird sowohl darauf abgestellt, dass das Verständnis des Prozessrechts im Verwaltungsrecht stark vom materiellen Recht abhängt, ${ }^{33}$ als auch darauf, dass das materielle Recht sich vollständig erst aus seiner jeweiligen prozessrechtlichen Bedeutung erschließt; ${ }^{34}$ manche statuieren allgemein eine praktische Untrennbarkeit. ${ }^{35}$ Materielles Recht und Prozessrecht erläutern sich gewissermaßen gegenseitig.

31 Vgl. BVerfGE 80, 103 (107); Maurer, in: Detterbeck/Rozek/von Coelln (Hrsg.), S. 535.

32 In diesem Sinne Salzwedel, in: VDStRL (Hrsg.), S. 15 (20): Kenntnis der machinery of justice; Berkemann, in: ZDRW 8 (2021), S. 224 (246).

33 Stern/Blanke, Verwaltungsprozessrecht in der Klausur, $\mathbb{~} 1$ Rn. 15.

34 Sauer, Examinatorium, Rn. $47 \mathrm{f}$.

35 Uerpmann-Wittzack, Examens-Repetitorium, Rn. 2; Franzius, in: ZDRW 2 (2015), S. 93 (103); Sauer, in: Krüper (Hrsg.), Rn. 20. Eine präzise Analyse von „Einwirkungen und Rückwirkungen“, aber auf der Grundlage einer vorgängigen „Entflechtung“, bei Reimer, in: DV 50 (2017), S. 395. 
Es lohnt sich, den Beispielen nachzugehen, die in dieser Hinsicht genannt werden. Sie knüpfen an einige der üblichen prozessualen Stationen an. So wird schon die Eröffnung des Rechtsweges als Beispiel für die Verzahnung genannt, mit Blick auf die Abgrenzung öffentlich-rechtliches und privatrechtliches Handeln. ${ }^{36}$ Aber genau genommen zeigt sich an dieser Stelle nur die verwaltungsprozessuale Konsequenz eines materiell-rechtlichen Befundes: Welche Rechtsnatur hat der geltend gemachte Anspruch? Zu beantworten ist also zunächst, wie im Zivilprozess, eine rein materiell-rechtliche Frage. Nach meinem Eindruck geht bei der Frage der Rechtswegeröffnung die eigentliche Struktur des Zusammenhangs mit dem materiellen Recht eher verloren, wenn der „klassische“ Streit um die Bedeutung des Begriffs der öffentlich-rechtlichen Streitigkeit nach $\mathbb{4} 40$ Abs. 1 VwGO übermäßig betont wird.

Des Weiteren wird vorgebracht, die Rechtsschutzform, d. h. die Klageart, sei abhängig von der Handlungsform. ${ }^{37}$ Aber auch hier ist der Zusammenhang wiederum nicht wirklich als Verzahnung greifbar. Betroffen ist das Klagebegehren, das seinerseits eine durchaus komplexe Angelegenheit darstellt. Nicht die Handlungsform als solche entscheidet über die Klageart, sondern das faktische Interesse einer Person an einem bestimmten Handeln der Verwaltung (während das subjektive öffentliche Recht den rechtlichen Schutz dieses Interesses betrifft). Wer sich „gegen“ einen versagenden Verwaltungsakt wendet, begehrt den Erlass eines begünstigenden Verwaltungsakts und erhebt die Verpflichtungsklage, wer einen belastenden Verwaltungsakt aufheben lassen möchte, erhebt die Anfechtungsklage. Das Interesse, nicht die Verwaltungsaktqualität entscheidet. Im Übrigen ist im Zusammenhang mit den Klagearten die Lage im Zivilrecht nicht grundsätzlich eine andere; allenfalls das Portfolio der zur Verfügung stehenden Klagearten ist etwas kleiner.

Der gleiche Befund - bloße Konsequenz - gilt für den Hinweis darauf, dass bei der Frage des richtigen Beklagten nach $\mathbb{\$} 78$ VwGO das Verwaltungsorganisationsrecht relevant sei. ${ }^{38}$ Die Relevanz besteht aber wiederum allein darin, dass die materielle Rechtslage gewisse prozessuale Konsequenzen hat. Bei genauerer Betrachtung löst sich hier sogar das Prozessrecht vom materiellen Recht, nämlich bei der passiven Prozessführungsbefugnis, die nach zutreffender, aber umstrittener Auffassung gerade durch $\mathbb{} 78$ VwGO geregelt wird. ${ }^{39}$ Die Autonomie der prozessualen Regelung bleibt verdeckt, wenn vorschnell das materielle Recht, in diesem Fall das Organisationsrecht, herangezogen wird. Die Unterschiede zwischen Prozessführungsbefugnis und Sachlegitimation, aktiv wie passiv, lassen sich nach meiner Erfahrung selbst in der Examensvorbereitung den Studierenden nur schwer vermitteln, und dies deshalb, weil kein hinreichendes Verständnis für das Prozessrecht und sein Zusammenspiel mit dem materiellen Recht besteht.

36 Sauer, Examinatorium, Rn. 49. Im gleichen Sinne Schwerdtfeger, in: JuS 1969, S. 472 (473).

37 Sauer, Examinatorium, Rn. 49.

38 Sauer, Examinatorium, Rn. 49.

39 Vgl. W.-R. Schenke, in: Kopp/Schenke, VwGO, $\mathbb{S} 78$ Rn. 1 m.w.N. 
Ein weiterer Anknüpfungspunkt für die Verzahnungsthese ist die Figur des subjektiven öffentlichen Rechts. „Die Frage, ob eine bestimmte gesetzliche Vorschrift ein subjektives öffentliches Recht gewährt, hat - auch in der Fallbearbeitung - vor allem einen prozessrechtlichen Hintergrund.“, so heißt es im verbreiteten Lehrbuch von Detterbeck..$^{40}$ Denn die Begründetheit der Anfechtungs- sowie der Verpflichtungsklage setze nach $\mathbb{S} 113$ Abs. 1 Satz 1 sowie $\$ 113$ Abs. 5 VwGO voraus, dass ein Recht verletzt sei. Aber eine solche Darstellung greift zu kurz. Sie vernachlässigt den Umstand, dass beide Klagen das Bestehen von Ansprüchen voraussetzen, die gegen die Verwaltung bestehen (dazu noch später). Damit stutzt sie die Rechtsstellung des Bürgers auf die eines Untertans zurück, der darauf angewiesen ist, Übergriffe des Staates vor Gericht abzuwehren, gegenüber der Verwaltung aber rechtlos ist. Dass ein subjektives öffentliches Recht in erster Linie eine Rechtsposition ist, die von der Verwaltung zu achten ist, geht verloren.

Eine Variante dieser verkürzenden Sicht besteht darin, die Bedeutung des subjektiven öffentlichen Rechts mit der Klagebefugnis nach $\$ 42 \mathrm{Abs.} 2 \mathrm{VwGO}$ zu verbinden. So stellen Voßkuble/Kaiser die Frage nach dem Bestehen eines subjektiven öffentlichen Rechts ausgehend davon, ob im Sinne des $\$ 42$ Abs. 2 VwGO eine Rechtsverletzung möglich ist. ${ }^{41}$ Nach Sauer wird die materielle Frage nach dem subjektiven öffentlichen Recht in Klausuren eigentlich nur im Rahmen der Zulässigkeitsvoraussetzungen relevant. ${ }^{42}$ Zutreffend sind solche Darstellungen allein vor dem Hintergrund, dass es über die - auf der Elfes-Konstruktion beruhende Adressatentheorie in der Begründetheitsprüfung der Anfechtungsklage in vielen Fällen ausreicht, die Rechtmäßigkeit des Verwaltungsakts zu prüfen. ${ }^{43}$ Denn sofern ein Verwaltungsakt in ein Grundrecht eingreift - und auf die Rechtfertigung dieser Annahme zielt die Adressatentheorie -, folgt aus der Rechtswidrigkeit des Verwaltungsakts logisch, dass der Verwaltungsakt ein subjektives öffentliches Recht verletzt. Aber als gedankliche Voraussetzung der Begründetheit der Klage bleibt das Erfordernis der Rechtsverletzung relevant. Bei der Anfechtungsklage ist dem verletzten Recht im Falle einer Drittanfechtung oder eines Ausschlusses des Aufhebungsanspruchs in der Begründetheit ohnehin mehr Aufmerksamkeit zuzuwenden. Erst recht kommt es bei den Leistungsklagen auf Ansprüche an.

Es scheint insofern ein eigentümlicher geistiger Mechanismus am Werk zu sein. In der eingeschliffenen gedanklichen Prüfung der Erfolgsaussichten der Klage ist das subjektive öffentliche Recht in der Klagebefugnis, die als Sachentscheidungsvoraussetzung vorrangig ist, „zuerst“ relevant. Die zitierten Äußerungen schreiben aber der daraus folgenden Anordnung von Prüfungsschritten ein Eigenleben zu. Sie erzeugen den Eindruck, die Gestalt des Prozesses hänge vom Vorliegen der Klagebefugnis ab. Was nicht stimmt: Ein verwaltungsgerichtliches Verfahren, das durch

40 Detterbeck, Allgemeines Verwaltungsrecht, Rn. 410. Im gleichen Sinne Voßkuble/Kaiser, in: JuS 2009, S. 16 (17).

41 Voßkuble/Kaiser, in: JuS 2009, S. 16 (17).

42 Sauer, Examinatorium, Rn. 49, 71.

43 Näher und m. w. N. Funke, Falldenken, Rn. 138, 226. 
eine Klage in Gang gesetzt wird, die wegen fehlender Klagebefugnis unzulässig ist, unterscheidet sich im Ablauf nicht von einem Verfahren, bei dem die Klage wegen fehlender Rechtsverletzung unbegründet ist. ${ }^{44}$ Die mündliche Verhandlung entfällt nicht, es gelten keine besonderen Entscheidungsfristen usw. Popularklagen lassen sich nicht verhindern, nur anders aburteilen.

Gewisse Berührungen von materiellem Recht und Prozessrecht resultieren fraglos aus den prozessualen Regelungen über die Klageerhebung. So wird zur Stützung der Verzahnungsthese auf das Institut der formellen Bestandskraft von Verwaltungsakten verwiesen. ${ }^{45}$ Nach dem Ablauf der Rechtsbehelfsfristen könne sich der Bürger nicht mehr darauf berufen, dass der Verwaltungsakt rechtswidrig sei. In der Tat ist es hilfreich für das Verständnis der Rechtsform des Verwaltungsakts, sich diese Zusammenhänge vor Augen zu führen. Aber bei genauerer Betrachtung sind sie recht kompliziert. Die formelle Bestandskraft ist ein rein prozessuales Institut: Sie bezeichnet nichts anderes als eben die Unanfechtbarkeit. Ob ihr Eintritt den materiell-rechtlichen Aufhebungsanspruch erlöschen lässt oder ihm nur die Durchsetzbarkeit nimmt, ist umstritten. ${ }^{46}$ Sofern man, was meines Erachtens zutreffend ist, auf die fehlende Durchsetzbarkeit abstellt, handelt es sich dabei um die verfah-

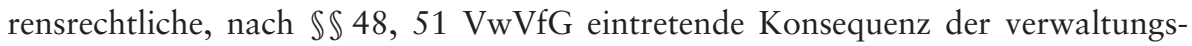
prozessualen Regelungen über die Klagefrist. Und selbst wenn hier engere Verknüpfungen angenommen werden sollten - warum dieser eine Schnittpunkt von materiellem Recht und Prozessrecht das integrative Format als solches rechtfertigen soll, ist nicht einsehbar.

Nicht einfach zu beurteilen ist die Rolle der Folgen, die die Erhebung der Klage bzw. des Widerspruchs nach $\mathbb{\$} 80$ Abs. $1 \mathrm{VwGO}$ hat. Ohne Zweifel ist es für das Verständnis des Verwaltungsakts als Handlungsform wichtig, die Vollziehbarkeit und in diesem Zusammenhang die aufschiebende Wirkung eines Rechtsbehelfs zu kennen. Aber die aufschiebende Wirkung ist ein prozessuales Institut, das das materielle Recht, auch das Verwaltungsverfahrensrecht (schon aus kompetenziellen Gründen), unberührt lässt. Die aufschiebende Wirkung soll den der Anfechtungsklage zu Grunde liegenden materiell-rechtlichen Aufhebungsanspruch vorprozessual sichern, und sie lässt den Inhalt der Regelung, die der Verwaltungsakt enthält, unberührt. ${ }^{47}$ Auch hier also steht im Ergebnis keine Verzahnung, sondern nur ein komplexes Zusammenspiel.

\section{Die starke Fassung}

Die schwache Fassung der Verzahnungsthese kann mithin nicht überzeugen. Die starke Fassung entstammt dem Problemkreis der Dichte verwaltungsgerichtlicher

44 Herausgearbeitet von Gierth, in: DÖV 1980, S. 893.

45 Schwerdtfeger/Schwerdtfeger, Öffentliches Recht in der Fallbearbeitung, $\mathbb{} 1 \mathrm{Rn} .4$; zum Folgenden auch Berkemann, in: ZDRW 8 (2021), S. 224 (238).

46 Dazu und zum Folgenden Funke, Falldenken, Rn. $231 \mathrm{ff}$.

47 Schoch, in: ders./Schneider, VwGO, $\mathbb{S} 80$ Rn. $104 \mathrm{f}$. 
Kontrolle im Verhältnis zu den Verwaltungsbehörden. Den Ausgangspunkt bilden die materiell-rechtlichen Lockerungen der Gesetzesbindung der Verwaltung: Ermessen, Abwägung, Beurteilungsspielraum. Diese Lockerungen sollen „verwaltungsprozessuale Folgen" haben, die daraus resultierten, dass die verwaltungsgerichtliche Kontrolle begrenzt sei, wenn die Verwaltung kraft der genannten Lockerungen die „Letztentscheidung“ treffe. ${ }^{48}$ In der Tat kann insofern durchaus eine Verzahnung diagnostiziert werden. Sie betrifft allerdings nicht den einzelnen Fall und seine Lösung, sondern die institutionelle Perspektive auf "die“ Verwaltung und auf „die“ Gerichtsbarkeit als staatliche Gewalten.

Die rechtsstaatlich gebotene Kontrolle der Verwaltung kann von den Verwaltungsgerichten stets nur im Rahmen ihres Auftrages zum Individualrechtsschutz (sowie ausdrücklicher weiterer gesetzlicher Aufträge) wahrgenommen werden. ${ }^{49}$ Lockerungen der Gesetzesbindung öffnen der Verwaltung Räume eigenständigen Entscheidens, in die auch die subjektiven Rechte der Einzelnen nicht hineinreichen. Ein insofern „beschränkter“ Individualrechtsschutz reduziert zwangsläufig das Kontrollpotential der Verwaltungsgerichte. Lockerungen der Gesetzesbindung werfen damit grundsätzliche verfassungsrechtliche Fragen auf; funktionale Erwägungen spielen eine Rolle, die sich aber wiederum verfassungsrechtlich legitimieren müssen. Wie bereits gesagt, wirken sich solche Erwägungen im konkreten Fall aber in der Regel nicht aus. Das Prozessrecht verhält sich dazu eigentlich nicht. $\$ 114$ Satz $1 \mathrm{VwGO}$ wiederholt für das Ermessen im Grunde nur, was $\mathbb{4} 40 \mathrm{VwVfG}$ statuiert. $^{50}$

Die starke Verzahnungsthese hat also ihre Berechtigung, ist aber für die Fallbearbeitung nicht unmittelbar relevant. Deshalb kann sie nicht als Stütze für das integrative Format dienen.

\section{Vor- und Nachteile des integrativen Formats}

\section{Vorteil: Vereinheitlichung und Standardisierung}

Es ist offensichtlich, dass eine einheitliche Ausrichtung am Lehr- und Prüfungsmuster „Zulässigkeit und Begründetheit" eines Rechtsbehelfs im Bereich des Öffentlichen Rechts eine hilfreiche Vereinheitlichung des Stoffes ermöglicht. Das Format hält den Stoff zusammen. Den Studierenden wird damit der Zugang zum Stoff und dessen Beherrschung erleichtert. Die Universitäten und Prüfungsämter können durch die damit ermöglichte Standardisierung von Aufgabenstellungen große Studierendenzahlen leichter bewältigen. Im Massenfach Rechtswissenschaft ist das ein nicht zu unterschätzender Umstand. Zugleich wird - zunächst - die Komplexität des Stoffes reduziert. Funktional leistet im Strafrecht die Strafbarkeitsprüfung (in

48 Stern/Blanke, Verwaltungsprozessrecht in der Klausur, $\mathbb{1} 1$ Rn. 16.

49 Vgl. Schoch, in: GVwR III, $\$ 50$ Rn. 6.

$50 \$ 114$ Satz 1 VwGO wurde vom Gesetzgeber im Bewusstsein des materiell-rechtlichen Charakters der Regelung nur in die VwGO aufgenommen, um eine Regelungslücke zu vermeiden, siehe BT-Drs. $3 / 55$, S. 32 . 
der Grundform der Prüfung: Tatbestand/Rechtswidrigkeit/Schuld) diese Vereinheitlichung, im Zivilrecht die Anspruchsmethode. ${ }^{51}$ Doch ist die ordnende Kraft des integrativen Formats, im Vergleich dazu, gering, weil es nicht materiell-rechtlich operiert.

Eine Grenze des Vereinheitlichungspotentials, das mit dem integrativen Format verbunden ist, bilden die staatshaftungsrechtlichen Schadenersatz- und Entschädigungsansprüche. Sie werden üblicherweise ohne prozessuale Einkleidung nach der zivilrechtlichen Anspruchsmethode gelehrt. Der pragmatische Grund für den Verzicht auf die Klageseite ist die - praktisch zweifellos fragwürdige - Rechtswegtrennung. Die Ansprüche sind den ordentlichen Gerichten zugewiesen, Art. 34 Satz 3 GG, Art. 14 Abs. 3 Satz 2 GG, $\mathbb{} 40$ Abs. 2 VwGO. Die Anspruchsmethode kommt schlicht deshalb zur Anwendung, weil es sich um Ansprüche handelt.

\section{Nachteile}

Die Komplexitätsreduktion, die mit der Vereinheitlichung kraft Integration von materiellem Verwaltungsrecht und Verwaltungsprozessrecht verbunden ist, hat beträchtliche Kosten, und es besteht die Gefahr einer übermäßigen Vereinfachung. Der meines Erachtens wohl (ge)wichtigste Nachteil des integrativen Formats ist, dass es einer konsequenten Dogmatisierung des Stoffes entgegensteht. Dieser Befund mag erstaunen, gilt doch die Nachkriegszeit als die Zeit der Verfeinerung der dogmatischen Methode in der Tradition Otto Mayers. ${ }^{52}$ Aber es ist nicht zu übersehen, dass, schon in der akademischen Verwaltungsrechtswissenschaft und erst recht in der lehrorientierten Literatur, erhebliche dogmatische Baustellen bestehen. Im Einzelnen sind diese Baustellen an dieser Stelle nicht aufzuzählen. ${ }^{53}$

Spiegelbildlich zu dieser Unterbilanz ist das integrative Format aber, zweitens, auch dem Verständnis des Verwaltungsprozessrechts nicht förderlich. In der Auseinandersetzung mit der schwachen Fassung der Verzahnungsthese deutete sich dies schon an (siehe C I). Mit dem integrativen Format lernen die Studierenden zwar gewisse Grundelemente des Prozessrechts kennen, und sie können diese in der Klausur anwenden. Doch nur den wenigsten Studierenden gelingt es, wirklich einen Sinn für prozessuale Institute oder gar für die Dynamik des prozessualen Geschehens zu entwickeln. Das Gerichtsverfahren wird ihnen als steriler Rechenapparat präsentiert. Natürlich kommt die universitäre Ausbildung hier an ihre Grenzen. Aber hilflos ist sie nicht: Chancen bietet etwa eine akademisch begleitete Prozesserprobung. ${ }^{54}$

51 Für das Strafrecht vgl. Roxin/Greco, Strafrecht AT, Bd. 1, $\mathbb{5} 7$ Rn. 4 u. 39; für das Zivilrecht Medicus, in: AcP 174 (1974), S. 313 (zum Weiterentwicklungspotential: Hofmann, in: JuS 2018, S. 833).

52 So jedenfalls Bumke, in: Schmidt-Aßmann/Hoffmann-Riem (Hrsg.), S. 73.

53 Verwiesen sei hier nur auf Funke, in: DV 52 (2019), S. 239; mit anderer Akzentuierung Reimer, in: DV 50 (2017), S. 395. Zum Stellenwert der Dogmatik im Verwaltungsrecht siehe die umfassende Bestandsaufnahme bei Kahl, Wissenschaft, Praxis und Dogmatik.

54 Interessant sind deshalb Gerichtssimulationen, dazu Funke/Eichinger, in: ZDRW 4 (2017), S. 135. 
Ein dritter Nachteil des integrativen Formats ist, dass es den Studierenden Verständnis für die praktisch wichtige gestaltende Rolle des Verwaltungsverfahrens nicht vermitteln kann. Die Studierenden lernen zwar das Verwaltungsverfahren in der Vorlesung zum Allgemeinen Verwaltungsrecht kennen. Aber die entscheidende Rolle, die dem Verfahren für die Konkretisierung der Rechte und Pflichten im Verhältnis von Verwaltung und Bürger zukommt, ${ }^{55}$ bleibt ihnen verborgen. In den Klausuren spielt das Verfahren nur noch ausschnitthaft eine Rolle, meistens unter dem Aspekt der formellen Rechtmäßigkeit eines Verwaltungsakts, wobei dann wiederum in der Regel nur die Anhörung nach $\$ 28 \mathrm{VwVfG}$ eine Rolle spielt. Teilweise wird sogar gelehrt, dass im Falle einer unterbliebenen Anhörung einfach unterstellt werden könne, der Fehler sei durch Nachholung nach $\mathbb{} 45$ Abs. 1 Nr. 3 VwVfG geheilt worden. ${ }^{56}$ Der rechtsstaatliche Sinn der Anhörung löst sich damit auf. Lehrbücher wie diejenigen von Martens über die „Praxis des Verwaltungsverfahren“ oder von Weides über „Verwaltungsverfahren und Widerspruchsverfahren“, die das Verwaltungsverfahren in den Mittelpunkt rücken, werden heute nicht mehr geschrieben. Viele für die Praxis wichtige Fragen fallen in der Lehre weg: Wer ist Beteiligter, welche Schritte muss die Behörde wählen, an wen richtet sich die Entscheidung etc.? Ähnlich ist der Befund für das Verwaltungsorganisationsrecht. Das integrative Format muss das Organisationsrecht auf das Verfahren zur Ermittlung der zuständigen Behörde reduzieren, relevant im Rahmen der Passivlegitimation sowie der formellen Rechtmäßigkeit eines Verwaltungsakts. Als Handlungssubjekt kommt die Verwaltung nicht in den Blick, ganz zu schweigen von der mit der Verwaltungsorganisation verknüpften Strukturierung und Rationalisierung staatlichen Handelns.

\section{E. Alternativen: insbesondere auf der Grundlage des Anspruchsmodells des Verwaltungsrechts}

Eine denkbare Alternative zum gegenwärtigen Zustand wäre die Strategie der Verbreiterung, im Sinne einer größeren Vielfalt von Aufgabenstellungen. Über die Wiedereinführung von Themenarbeiten soll hier nicht spekuliert werden. Für die Bearbeitung von Fällen kennt die Ausbildungsliteratur zahlreiche Fragestellungen. ${ }^{57}$ Jedoch ist es schwierig, eine solche Vielfalt unter eine leitende Idee zu stellen. Es besteht die Gefahr, dass der Stoff zerfasert. Die nötige Einheitlichkeit könnte allenfalls dann erreicht werden, wenn Klausuren konsequent als „Rechtsaktklausur" ${ }^{58}$ ausgestaltet werden, bei der allein die Rechtmäßigkeit eines staatlichen Rechtsakts zu prüfen ist. Aber diesem Format fehlt gewissermaßen die

55 Siehe nur Brüning, in: DV 48 (2015), S. 155 (167f.).

56 Brinktrine/Kastner, Fallsammlung, S. 35; Becker/Heckmann u.a., Klausurenbuch, S. 112. Kritisch gegenüber dieser Strategie Uerpmann-Wittzack, Examens-Repetitorium, Rn. 201a.

57 Butzer/Epping, Arbeitstechnik, S. $14 \mathrm{ff}$. Interessant ist die Vielfalt der Aufgabenstellungen in Österreich; das Verwaltungsverfahren kommt darin mehr zur Geltung als in Deutschland (Müllner/Vašek, Casebook; Bachmann (Hrsg.), Besonderes Verwaltungsrecht; Müller/Wallnöfer/Wimmer (Hrsg.), Fallbuch).

58 Hierzu Lindner, Öffentliches Recht, Rn. 53 ff. 
Bodenhaftung. Staatliches Handeln muss hier aus seinem von Handlungen und Interessen geprägten Kontext herausgelöst werden. Dem Verständnis ist das nicht zuträglich.

Meines Erachtens sprechen gute Gründe dafür, nicht in die Breite, sondern gewissermaßen in die Tiefe zu gehen. Lehre und Prüfungen sollten sich stärker als bisher an einem verwaltungsrechtlichen Denken orientieren, dass als Anspruchsmodell bezeichnet werden kann. ${ }^{59}$ Dieser Weg begegnet den Nachteilen, die mit dem gegenwärtig praktizierten integrativen Format verbunden sind, ohne dessen Vorteil, die Vereinheitlichung, zu verschenken. Aber die Nachteile, die er haben könnte, bedürfen der Diskussion.

\section{Was ist das Anspruchsmodell?}

„Das“ Anspruchsmodell gibt es freilich nicht. Nicht einmal jede an Ansprüchen orientierte Darstellung des öffentlichen Rechts bildet schon ein „Anspruchsmodell“ ${ }^{60}$ Unter diesem Begriff lassen sich im Detail durchaus unterschiedliche Ansätze versammeln, die eine charakteristische methodische Maßgabe verbindet und die in einer bestimmten verwaltungsrechtsdogmatischen Tradition stehen. ${ }^{61}$ Keineswegs ist es schon in jeder Hinsicht ausgearbeitet.

Die methodische Maßgabe stammt aus dem Zivilrecht: die „Anspruchsmethode“. Sie rückt die gerichtliche Durchsetzbarkeit von Ansprüchen in den Mittelpunkt und ist insofern „aktionenrechtlich“. Nach Medicus führt sie dabei „zu einer prozessförmigen - und zwar an der Leistungsklage orientierten - Aufgliederung des Stoffs". ${ }^{62}$ Diesen Gedanken macht das Anspruchsmodell für das Verwaltungsrecht fruchtbar. Die Rechtssätze des Verwaltungsrechts sollen „aktionenrechtlich“ dargestellt werden. Das meint freilich nicht die im Vorstehenden kritisierte Verzahnung der verwaltungsgerichtlichen Klagen mit dem materiellen Verwaltungsrecht, womöglich im Sinne einer Ableitung von subjektiven Rechten aus den Klagemöglichkeiten, sondern eine Trennung. Wie im Zivilrecht ist der Anspruch materieller Natur. Jedoch wahrt die Ablösung des materiellen Verwaltungsrechts von den Klagen insofern den Bezug zum Verwaltungsprozess, als die klageförmige Durchsetzbarkeit des Rechts als Wegweiser für die Strukturierung rechtlichen Materials dient.

Sicherlich lässt die Anspruchsmethode im Verwaltungsrecht Raum für verschiedene konkretisierende Ansätze. Am ergiebigsten und im Übrigen auch am dominantesten ist meines Erachtens ein Traditionsstrang verwaltungsrechtlichen Denkens, der auf Georg Jellinek zurückgeht und der sich an den Potentialen einer möglichst präzisen Analyse von subjektiven öffentlichen Rechten orientiert. Aus der Zeit

59 Der treffende Begriff nach der kritischen Abhandlung von Buchheim, Actio, Anspruch, subjektives Recht. Gegenkritik bei Funke, in: DV 52 (2019), S. 239.

60 Dies gilt etwa für Thieme/Bull, in: JZ 1969, S. 422; Frenz, Öffentliches Recht.

61 Ausführlich Funke, Falldenken, Rn. 17 ff.

62 Vgl. Medicus, in: AcP 174 (1974), S. 313 (331). 
der frühen Bundesrepublik stammen wichtige Arbeiten von Hans J. Wolff und Hans Heinrich Rupp, aus jüngerer Zeit etwa von Wolf-Rüdiger Schenke. ${ }^{63} \mathrm{In}$ der Ausbildungsliteratur ist die Tradition ansatzweise bei Lindner präsent. ${ }^{64}$

\section{Denkbare Aufgabenstellungen}

Das Anspruchsmodell reduziert verwaltungsrechtliche Fälle keineswegs auf eine Anspruchsprüfung. ${ }^{65}$ Es rückt zunächst einmal die verwaltungsrechtlichen Rechtsverhältnisse in den Mittelpunkt. Rechte wie auch Ansprüche sind dann relevant, aber auch Verpflichtungen. Auf dieser Grundlage sind in Lehre und Prüfung ganz verschiedene Aufgabenstellungen denkbar. Sie lassen sich danach ordnen, ob sie eine Aktionsperspektive oder eine Reaktionsperspektive einnehmen. Kombinationen dieser Perspektiven sind selbstverständlich denkbar.

\section{Aktionsperspektive}

In der Aktionsperspektive schlummern beträchtliche erkenntnisfördernde Potentiale. Sie orientiert sich daran, dass es gar nicht erst zu einer Rechtsverletzung kommen soll. In den Blick rückt dann zum einen das ursprüngliche Handeln der Einzelnen in Anbetracht der Verwaltungsrechtssätze. Es kann zum Gegenstand einer Aufgabenstellung gemacht werden: Darf A bauen? Muss B dem Platzverweis Folge leisten, verletzt (oder verletzte) der Platzverweis B's Rechte? Muss C die Vollstreckung dulden? Zum anderen sind Fragen denkbar, die auf die behördliche Entscheidung abstellen: ${ }^{66}$ Darf die Behörde in einer Gefahrsituation einschreiten? Muss die Behörde den Verwaltungsakt zurücknehmen? Hierzu zählen auch die praktisch wichtigen Konstellationen, in denen der Einzelne Ansprüche geltend macht, die unmittelbar gesetzlich, vertraglich oder durch Verwaltungsakt begründet sind (d. h. nicht aus einer Rechtsverletzung resultieren), beispielsweise auf Erteilung einer Genehmigung oder auf Erstattung rechtsgrundloser Leistungen.

Während das damit verbundene Prüfprogramm vertraut ist, erscheinen in der Aktionsperspektive andere Konstellationen in einem neuen Licht. Sofern behördliche Ermessensspielräume bestehen, ist die rechtliche Prüfung darauf beschränkt, diesen Spielraum konkret abzustecken, einschließlich der Anforderungen an ein verhältnismäßiges Handeln. Es spricht aber auch nichts dagegen, Zweckmäßigkeitserwägungen zuzulassen, sofern sie in der Bearbeitung als solche gekennzeichnet werden. Das im Polizei- und Ordnungsrecht relevante „Recht auf Einschreiten“ ist nicht erst anhand der Frage nach der Abwehr der behördlichen Entscheidung, nicht einzuschreiten, zu behandeln, sondern muss unmittelbar auf die Entscheidungssituati-

63 Nachweise bei Funke, Falldenken, Rn. 5; siehe zudem Wolff/Bachof u.a., Verwaltungsrecht I, $\mathbb{3} 32$, $\mathbb{S} \mathbb{S} 40-43$.

64 Lindner, Öffentliches Recht, Rn. $1203 \mathrm{ff}$.

65 Dieser Abschnitt entwickelt Überlegungen weiter, die sich bei Funke, Falldenken, Rn. 395, finden.

66 Insofern stehen hier rechtsakt- und verhaltensbezogene Perspektive nebeneinander, allerdings nicht im Sinne eines steuerungswissenschaftlichen Verständnisses, dazu Bumke, in: Schmidt-Aßmann/ Hoffmann-Riem (Hrsg.), S. 73 (127). 
on der Behörde bezogen werden. Das Recht auf fehlerfreie Ermessensentscheidung gewinnt an Profil, die Abgrenzung von Ermessens- und Zweckmäßigkeitserwägungen steht unter einem anderen Vorzeichen.

Die Aktionsperspektive erlaubt es des Weiteren, das Verwaltungsverfahren und die Stellung, die der Einzelne darin hat, stärker in den Blick zu nehmen (siehe unter D. II.), bis hin zur eigentlich ganz schlichten, aber unterbelichteten Frage, ob und inwieweit eine Antragstellerin einen Anspruch auf Bescheidung des Antrages hat. ${ }^{67}$

Prozessual kann die Aktionsperspektive, je nach betroffener Rechtsposition, mit den Leistungsklagen oder mit der Feststellungsklage nach $\mathbb{} 43 \mathrm{VwGO}$ verknüpft werden. In der Verpflichtungsklage erfolgt, soweit sie auf ein Vornahmeurteil gerichtet ist, gewöhnlich ohnehin eine Anspruchsprüfung. Schwierig ist insofern allerdings die Lage beim Recht auf fehlerfreie Ermessensentscheidung, was hier nicht weiter zu erörtern ist. ${ }^{68}$ Die Feststellungsklage ist nicht auf die Durchsetzung von materiellen Ansprüchen gerichtet, auch nicht auf die Durchsetzung eines gegen die Verwaltung gerichteten Feststellungsanspruchs. Es handelt sich bei ihr, wie im Zivilprozess, um ein rein prozessuales Institut.

\section{Reaktionsperspektive}

Die Reaktionsperspektive ist zum einen aus der Anfechtungsklage vertraut, zum anderen aus den staatshaftungsrechtlichen Leistungsklagen. Sie betrachtet sozusagen das, was die „Begründetheit“ dieser Klagen ausmacht, strikt im Verhältnis von Verwaltung und Bürger/-in. Es kommt allein auf eine materiell-rechtliche Prüfung der in diesem Verhältnis angesiedelten Ansprüche an.

Das hat verschiedene Vorteile und ist an sich nichts Neues. Schwer ist nur die konsequente Durchführung des damit verbundenen Programms. Es gibt zu denken, wenn Ule die Frage danach, ob es im Öffentlichen Recht, so wie im Zivilrecht, um Ansprüche geht, in einer gewundenen Darlegung zuerst verneint und dann bejaht. ${ }^{69}$ Für die Aktionsperspektive ist die Anspruchsprüfung, wie dargelegt, anerkannt. Etwas komplexer liegen die Dinge bei reaktionsrechtlichen Ansprüchen, die mit der allgemeinen Leistungsklage durchgesetzt werden. Hier wird häufig der Folgenbeseitigungsanspruch in den Vordergrund gerückt, was die Zusammenhänge mit anderen reaktionsrechtlichen Ansprüchen (Unterlassungs- und Beseitigungsansprüche) in den Hintergrund treten lässt. ${ }^{70}$ All diese Ansprüche können für die Fallprüfung einen einheitlichen Rahmen bilden. Auch die Konstellation des „Rechts auf Einschreiten“ fällt darunter, nun unter dem Gesichtspunkt des Anspruchs darauf, dass die fehlerhafte Entscheidung, nicht einzuschreiten, aufge-

67 Siehe dazu Funke, Falldenken, Rn. 114.

68 Näher Funke, Falldenken, Rn. $337 \mathrm{ff}$.

69 Ule, in: JuS 1961, S. 190 (191 f.).

70 Siehe nur Maurer/Waldhoff, Allgemeines Verwaltungsrecht, $\$ 30 ;$ Baldus/Grzeszick/Wienhues, Staatshaftungsrecht, $\mathbb{S} 1$. Anders hingegen der grundrechtliche Schutzanspruch auf Unterlassung, Beseitigung und Herstellung bei Ossenbühl/Cornils, Staatshaftungsrecht, 7. Teil. 
hoben wird (u. U. kann zugleich in der Aktionsperspektive das „ursprüngliche“ Recht auf Einschreiten wieder geltend gemacht werden).

Der Problemfall für eine reine Anspruchsprüfung entsteht in erster Linie in den Konstellationen, die im integrativen Format von der Anfechtungsklage abgedeckt werden. Zwar ist theoretisch anerkannt, dass die Klage der Durchsetzung eines materiell-rechtlichen Aufhebungsanspruchs dient. ${ }^{71}$ Dieser Anspruch wird in $\$ 113$ Abs. 1 VwGO vorausgesetzt, nicht begründet. Aber die Konsequenzen, die das Bestehen dieses Anspruchs hat, sind schon in der Wissenschaft kontrovers, und in der Ausbildungsliteratur ist er kaum präsent. Aufgabenstellungen, die darauf gerichtet sind, einen gegen die Verwaltung gerichteten Anspruch auf Aufhebung eines rechtsverletzenden Verwaltungsakts zu prüfen, und zwar ohne Klage, stiften enorme Verwirrung - unter Studierenden wie auch unter wissenschaftlichen Mitarbeiter/-innen. ${ }^{72}$ Das liegt auch daran, dass die verwaltungsverfahrensrechtliche Seite des Aufhebungsbegehrens regulatorisch unterbelichtet ist. Ein gesondertes Aufhebungsverfahren gibt es nicht. Häufig rückt dann $\mathbb{} 48 \mathrm{VwVfG}$ in den Blick, aber nicht als Verfahrensregelung, sondern als denkbare Grundlage des Aufhebungsanspruchs. Nur: Jenseits des Verfahrens regelt die Norm materiell-rechtlich nichts. Ihr Telos, als verfahrensrechtliche Regelung die Verwaltung zu Gunsten des Bürgers an einmal ergangene Entscheidungen zu binden („materielle Bestandskraft“), ist den Studierenden bzw. Mitarbeiter/-innen nur mit größter Mühe zu vermitteln. Zwar ist umstritten, inwiefern die missglückte Textfassung des $\$ 48$ Abs. $1 \mathrm{VwVfG}$ die Aufhebung rechtsverletzender Verwaltungsakte vor dem Eintritt der Unanfechtbarkeit in das Ermessen der Verwaltung stellt. ${ }^{73}$ Aber zur Lehrpraxis steht dieser Streit in weiter Entfernung. ${ }^{74}$

\section{Vor- und Nachteile des anspruchsbasierten Formats}

Es hat verschiedene Vorteile, das Verwaltungsrecht primär in der Form von Ansprüchen - der Einzelnen wie auch der Verwaltung - zu erfassen. Ein verwaltungsrechtliches Anspruchsmodell gewährleistet die auch im Zivilrecht mit der Anspruchsmethode verbundene Vereinheitlichung des Stoffes, nun auch unter Einbeziehung des Staatshaftungsrechts. Man kann diskutieren, ob die vereinheitlichende Funktion im Öffentlichen Recht mit Blick auf dessen hoheitlichen Charakter Einschränkungen erfahren muss. Es dürfte wenig ergiebig sein, polizeiliche Befugnisse in Ansprüche umzudeuten, aber darum geht es auch nicht.

71 Riese, in: Schoch/Schneider (Hrsg.), VwGO, \$113 Rn. 7 m. w. N.

72 So meine persönliche Erfahrung, nachdem ich mehrfach Klausuren dieser Art gestellt habe. Auch eine intensive Vorbereitung in den Propädeutischen Übungen (= Arbeitsgemeinschaften) stieß hier an Grenzen.

73 Vgl. Sachs, in: Stelkens/Bonk/Sachs (Hrsg.), VwVfG, $\$ 48$ Rn. 48 m. w. N.

74 Repräsentativ insofern Uerpmann-Wittzack, Examens-Repetitorium, S. 112 ff., der unter der Überschrift „Aufhebung von Verwaltungsakten außerhalb von Rechtsbehelfsverfahren“" u. a. $\$ 48$ VwVfG behandelt, den Aufhebungsanspruch aber mit keinem Wort erwähnt. 
Das anspruchsbasierte Format behandelt das Verwaltungsprozessrecht nur akzessorisch. Das war früher durchaus üblich (siehe unter B. I.). Lehrbuchdarstellungen können gemäß dem Format aufgebaut werden. ${ }^{75}$ Das gedankliche Vorgehen ist in der gegenwärtigen Praxis der Fallbearbeitung aber nicht wirklich anders. Nur wird es in Vorüberlegungen gepresst, die die „Marschroute“ für die prozessualen Stationen festlegen, ohne dass sie niederzuschreiben sind.

Die Entflechtung, ${ }^{76}$ die mit der akzessorischen Stufung einhergeht, erschließt der Lehre wichtige Potentiale. Sie öffnet einerseits den Weg zu einer konsequent dogmatischen Behandlung des materiellen Verwaltungsrechts und schafft andererseits die Bedingungen für eine klarere Behandlung des Prozessrechts. Die Dogmatisierung ist aber kein Selbstzweck. Sie ist nicht zuletzt wichtig für die Offenlegung wichtiger Wertungsebenen. ${ }^{77} \mathrm{Ihr}$ kommt zudem eine rechtsstaatlich-demokratische Funktion zu: Sie zwingt dazu, das Verwaltungsrecht vorrangig aus der Perspektive der Rechtsverhältnisse zwischen Bürger und Verwaltung zu denken. Damit ist verbunden, dass die den Ansprüchen zu Grunde liegenden Pflichten stärker hervortreten. Rechte sind von der Verwaltung zu achten, Ansprüche zu erfüllen. Eingriffe tragen nicht schon die Teleologie der Rechtfertigbarkeit in sich. Das sollte auch den Studierenden vermittelt werden; es geht aber unter in einer auf „Grundrechtsprüfung“ fokussierten Lehrpraxis. Die genannten Rechtsbindungen machen das Fundament der Rechtsstaatlichkeit des Grundgesetzes aus, mag auch der gerichtliche Rechtsschutz den vielgerühmten „Schlussstein“ im Gebäude des Rechtsstaates darstellen. Zugleich würde ein Zurücktreten des integrativen Formats erlauben, neben dem Rechtsschutz andere Instrumente zur Kontrolle der Verwaltung genauer in den Blick zu nehmen.

Freilich drängt sich ein Einwand auf: Ein Mehr an Dogmatik könne den Studierenden im Verwaltungsrecht gar nicht zugemutet werden. Das Anspruchsmodell belaste Lehre und Prüfung mit überflüssiger Komplexität. Aber eigentlich macht das Anspruchsmodell nur deutlich, wie komplex - und insofern wahrhaft anspruchsvoll - das Verwaltungsrecht tatsächlich ist. An dieser Stelle kommt es deshalb meines Erachtens zum Schwur: Wer an der Wissenschaftlichkeit des Rechtsstudiums festhalten möchte, darf keine Zugeständnisse an eine trivialisierende Lehrpraxis machen. ${ }^{78}$ Was wissenschaftlich richtig ist, muss den Studierenden zugemutet werden. Zudem ist zu berücksichtigen, dass ein Abrücken vom integrativen Format die Bedeutung des Verwaltungsprozessrechts schrumpfen lässt, was Raum dafür schafft, das materielle Recht gründlicher zu behandeln. Ins Gewicht fällt schließlich auch, dass das Anspruchsmodell naturgemäß strukturelle Anschlüsse an das Zivilrecht ermöglicht (Forderungsbegriff, Struktur des Schutzes von Rechten etc.), insofern also wiederum leichter zugänglich ist.

75 Ein Versuch bei Funke, Falldenken, Teil III; detailliert hierzu Berkemann, in: ZDRW 8 (2021), S. 224 (235 ff.)

76 Vgl. Fn. 35.

77 Dazu näher Funke, Falldenken, Rn. 391.

78 Anderer Ansicht ist wohl Berkemann, in: ZDRW 8 (2021), S. 224 (234f.). 


\section{F. Schluss}

Im Öffentlichen Recht ist die akademische Lehre und Prüfung ebenso wie das Staatsexamen von einer nahezu totalen Integration des Verwaltungsprozessrechts geprägt. Diese Praxis hat sich, nur von spärlicher Reflexion begleitet, eingestellt, ist aber einer rationalen Rekonstruktion nicht zugänglich. Es ist nicht klar, welche spezifische Befähigung im Studium des Verwaltungsrechts mit dem integrativen Format vermittelt werden soll. Die Beherrschung des Prozessrechts ist im Zivilrecht und im Strafrecht nicht mehr und nicht weniger wichtig. Aus guten Gründen wird im Zivilrecht und im Strafrecht darauf verzichtet, den Stoff integriert zu vermitteln. Eine „Verzahnung“ des materiellen und des Prozessrechts, die insofern eine Besonderheit des Öffentlichen Rechts darstellen könnte, besteht nicht.

Die prozessuale Einkleidung verwaltungsrechtlicher Fälle ist also verzichtbar. Das nackte Verwaltungsrecht muss sich nicht schämen. Mit der Verabschiedung des integrativen Formats droht dem Verwaltungsrecht nicht der Untergang in unübersehbarer Vielfalt. Ein anspruchsbasiertes Format, in dem das Prozessrecht strikt akzessorisch zum materiellen Recht behandelt wird, kann die nötige Vereinheitlichung und Standardisierung des Stoffes gewährleisten, und es hält den Weg zu einer (weiteren) konsequenten Dogmatisierung des Verwaltungsrechts offen.

\section{Literaturverzeichnis}

Achterberg, Norbert, Fälle und Lösungen nach höchstrichterlichen Entscheidungen: Allgemeines Verwaltungsrecht, Karlsruhe 1975.

Bachmann, Susanne u. a. (Hrsg.), Besonderes Verwaltungsrecht: Fälle und Lösungen, Wien 2020.

Baldus, Manfred/Grzeszick, Bernd/Wienhues, Sigrid, Staatshaftungsrecht, 5. Aufl., Heidelberg u. a. 2018.

Becker, Ulrich/Heckmann, Dirk/Kempen, Bernhard/Manssen, Gerrit, Klausurenbuch Öffentliches Recht in Bayern, 4. Aufl., München 2019.

Berkemann, Jörg, Vorüberlegungen und Strukturierung der verwaltungsrechtlichen Klausur, in: ZDRW 8 (2021), S. 224-249.

Böhm, Monika/Gaitanides, Charlotte, Fälle zum allgemeinen Verwaltungsrecht, 4. Aufl., München 2007.

Bretthauer, Sebastian/Colllin, Peter/Egidy, Stefanie/Spiecker Döhmann, Indra, 40 Klausuren aus dem Verwaltungsrecht, 12. Aufl., München 2020.

Brinktrine, Ralf/Kastner, Berthold, Fallsammlung zum Verwaltungsrecht, 2. Aufl., Berlin, Heidelberg 2005.

Brüning, Christoph, Verwaltung und Verwaltungsgerichtsbarkeit, in: Die Verwaltung 48 (2015), S. 155174.

Brüning, Christoph/Suerbaum, Joachim, Examensfälle zum Öffentlichen Recht, München 2005.

Buchbeim, Johannes, Actio, Anspruch, subjektives Recht. Eine aktionenrechtliche Rekonstruktion des Verwaltungsrechts, Tübingen 2017.

Bull, Hans Peter, „Angstfach“ Verwaltungsrecht. Was wir den Studenten zumuten, in: JZ 1998, S. 338343.

Bumke, Christian, Die Entwicklung der verwaltungsrechtswissenschaftlichen Methodik in der Bundesrepublik, in: Schmidt-Aßmann/Hoffmann-Riem (Hrsg.), Methoden der Verwaltungsrechtswissenschaft, Baden-Baden 2004, S. 73-130. 
Butzer, Hermann/Epping, Volker, Arbeitstechnik im öffentlichen Recht, 3. Aufl., Stuttgart 2006.

Detterbeck, Steffen, Allgemeines Verwaltungsrecht. Mit Verwaltungsprozessrecht, 19. Aufl., München 2021.

Dietlein, Johannes/Burgi, Martin/Hellermann, Johannes, Klausurenbuch Öffentliches Recht in Nordrhein-Westfalen, 3. Aufl., München 2021.

Eisentraut, Nikolas (Hrsg.), Fälle zum Verwaltungsrecht, Berlin u. a. 2020.

Eisentraut, Nikolas, Zur Arbeit mit diesem Buch, in: ders. (Hrsg.), Verwaltungsrecht in der Klausur, Berlin u. a. 2020, S. XXXVII-XXXVIII.

Englisch, Joachim/Cryns, Anna S., Fälle und Lösungen zum allgemeinen Verwaltungsrecht einschließlich Staatshaftungsrecht, Stuttgart 2008.

Erbel, Günter, Öffentlich-rechtliche Klausurenlehre mit Fallrepetitorium, Bd. 2 (Verwaltungsrecht), Köln 1981.

Fleiner, Fritz, Verwaltungsrechtsfälle zum akademischen Gebrauch, 2. Aufl., Tübingen 1919.

Franzius, Claudio, Struktur und Inhalte eines verwaltungsrechtlichen Curriculums, in: ZDRW 2 (2015), S. 93-107.

Frenz, Walter, Öffentliches Recht. Eine nach Anspruchszielen geordnete Darstellung zur Examensvorbereitung, 8. Aufl., München 2019.

Funke, Andreas, Ansprüche und Klagen im Verwaltungsrecht, in: Die Verwaltung 52 (2019), S. 239-257.

Funke, Andreas, Falldenken im Verwaltungsrecht, Berlin 2020.

Funke, Andreas, $\mathbb{} 15$ Rechtswissenschaftliches Grundlagencurriculum: Funktionen und Formen, in: Krüper (Hrsg.), Rechtswissenschaft lehren - Handbuch der juristischen Fachdidaktik, Tübingen 2022, S. 383-408.

Funke, Andreas, Georg Jellinek, System der subjektiven öffentlichen Rechte (1892), in: Brocker (Hrsg.), Geschichte des politischen Denkens: Das 19. Jahrhundert, Frankfurt am Main 2021, S. 762-775.

Funke, Andreas/Eichinger, Annette, Der Mock Trial im Verwaltungsprozessrecht. Zur Simulation einer Gerichtsverhandlung am Verwaltungsgericht, in: ZDRW 4 (2017), S. 135-144.

Gierth, Karl, Klagebefugnis und Popularklage, in: DÖV 1980, S. 893-898.

Hans, H[einrich]., Öffentliches Recht II (Verwaltungsrecht und Verwaltungsgerichtsverfahren). 75 Fälle mit Lösungen, Stuttgart u. a. 1960.

Hans, H[einrich]., Öffentliches Recht II (Verwaltungs- und Verfahrensrecht). 60 Fälle mit Lösungen, Stuttgart 1975.

Haferkamp, Hans-Peter, Rechtsfälle in der juristischen Ausbildung der Pandektenwissenschaft, in: ZRGG 138 (2021), S. 283-294.

Heinze, Arne-Patrik, Systematisches Fallrepetitorium Allgemeines Verwaltungsrecht (2 Bände), 2. Aufl., Berlin u. a. 2020.

Hoffmann-Riem, Wolfgang (Hrsg.), Sozialwissenschaften im öffentlichen Recht. Fälle und Lösungen in Ausbildung und Prüfung, Neuwied 1981.

Hofmann, Franz, „Anspruchsdenken“ und „Remedydenken“ im deutschen Privatrecht, in: JuS 2018, S. 833-838.

Jellinek, Georg, System der subjektiven öffentlichen Rechte, 2. Aufl., Tübingen 1905.

Kahl, Wolfgang, Wissenschaft, Praxis und Dogmatik im Verwaltungsrecht, Tübingen 2020.

Kern, Eduard/Widenmann, Gustav, Das juristische Studium in Baden-Württemberg und sein Abschluß durch die Referendarprüfung und die Promotion, Stuttgart 1958.

Klein, Karl Heinz, Gutachten und Urteil im Verwaltungsprozeß, Berlin Frankfurt a. M. 1967.

Kollath, Erich, Praktische Verwaltungsrechtsfälle mit Lösungen, Mannheim 1930. 
Kopp, Ferdinand/Schenke, Wolf-Rüdiger, Verwaltungsgerichtsordnung - Kommentar, 27. Aufl., München 2021.

Ksoll, Eberhard, Verwaltungsrechtsfälle und Klausuren mit Lösungen, Düsseldorf 1959.

Lindner, Josef Franz, Öffentliches Recht. Systematisches Lehrbuch zur Examensvorbereitung im Freistaat Bayern, 2. Aufl., Stuttgart 2017.

Lomfeld, Bertram (Hrsg.), Die Fälle der Gesellschaft. Eine neue Praxis soziologischer Jurisprudenz, Tübingen 2017.

Martens, Joachim, Die Praxis des Verwaltungsverfahrens, München 1985.

Maurer, Hartmut, Der Justizgewährungsanspruch, in: Detterbeck/Rozek/von Coelln (Hrsg.), Festschrift für Herbert Bethge zum 70. Geburtstag, Berlin 2009, S. 535-550.

Maurer, Hartmut/Waldhoff, Christian, Allgemeines Verwaltungsrecht, 20. Aufl., München 2020.

Mayer, Otto, Deutsches Verwaltungsrecht, Bd. 1, Berlin, 3. Aufl. 1924 (Nachdruck 1961).

Medicus, Dieter, Anspruch und Einrede als Rückgrat einer zivilistischen Lehrmethode, in: AcP 174 (1974), S. 313-331.

Muckel, Stefan, Fälle zum Besonderen Verwaltungsrecht, 7. Aufl., München 2019.

Müller, Thomas/Wallnöfer, Klaus/Wimmer, Andreas W. (Hrsg.), Fallbuch Verwaltungs- und Verwaltungsverfahrensrecht, 4. Aufl., Wien 2017.

Müllner, Josef/Vašek, Markus, Casebook Staats- und Verwaltungsrecht, 4. Aufl., Wien 2018.

Münch, Ingo von, Übungsfälle zum Staatsrecht, Verwaltungsrecht, Völkerrecht, Bad Homburg vor d. Höhe 1967.

Ossenbühl, Fritz/Cornils, Matthias, Staatshaftungsrecht, 6. Aufl., München 2013.

Peine, Franz-Joseph, Klausurenkurs im Verwaltungsrecht, 6. Aufl., Heidelberg 2016.

Redelberger, Oskar, Das öffentliche Recht. Fälle mit Lösungen, Berlin u. a. 1941.

Reimer, Philipp, Materielles Verwaltungsrecht und Prozessrecht - Einwirkungen und Rückwirkungen, in: Die Verwaltung 50 (2017), S. 395-420.

Ridder, Helmut/Schmidt, Walter, Über Klausuren im allgemeinen und eine öffentlich-rechtliche Referendarklausur im besonderen, in: JuS 1966, S. 237-243.

Rinken, Alfred, Einführung in das juristische Studium. Juristenausbildung und Juristenpraxis im Verfassungsstaat, 3. Aufl., München 1996.

Roxin, Claus/Greco, Luís, Strafrecht, Allgemeiner Teil, Bd. 1, 5. Aufl., München 2020.

Salzwedel, Jürgen, Empfiehlt sich eine Vorlesung „Einführung in das öffentliche Recht“, in: Vereinigung der Deutschen Staatsrechtslehrer (Hrsg.), Probleme des öffentlichen Rechts im Universitätsunterricht, Hamburg 1969, S. 15-21.

Sauer, Heiko, Examinatorium Allgemeines Verwaltungsrecht und Verwaltungsprozessrecht, Baden-Baden 2020.

Saner, Heiko, $\$ 18$ Struktur und Gegenstand des Verwaltungsrechtscurriculums, in: Krüper (Hrsg.), Rechtswissenschaft lehren - Handbuch der juristischen Fachdidaktik, Tübingen 2021 (i. E.).

Sauer, Heiko, Klausurtraining Allgemeines Verwaltungsrecht und Verwaltungsprozessrecht, 2. Aufl., Baden-Baden 2021.

Schmidt-Jortzig, Edzard/Ipsen, Jörn, 30 Klausuren aus dem Verwaltungsrecht mit Lösungsskizzen, Frankfurt/Main 1978.

Schneider, Hans, Öffentliches Recht (47 Fälle mit Lösungen), Leipzig 1938.

Schneider, Hans, Die Übungen für Anfänger und Vorgerückte im öffentlichen Recht, in: Vereinigung der Deutschen Staatsrechtslehrer (Hrsg.), Probleme des öffentlichen Rechts im Universitätsunterricht, Hamburg 1969, S. 28-33. 
Schoch, Friedrich, Übungen im Öffentlichen Recht II. Verwaltungsrecht und Verwaltungsprozeßrecht, Berlin 1992.

Schoch, Friedrich, $\mathbb{5 0}$ Gerichtliche Verwaltungskontrollen, in: Hoffmann-Riem/Schmidt-Aßmann/ Voßkuhle (Hrsg.), Grundlagen des Verwaltungsrechts, Bd. 3, 2. Aufl., München 2013, S. 743-1074.

Schoch, Friedrich/Schneider, Jens-Peter (Hrsg.), Verwaltungsrecht VwGO, Stand: Febr. 2021, München.

Schrödter, Hans, Die verwaltungsrechtliche Entscheidung. Eine Anleitung für Prüfung und Praxis mit Klausuren für die große Staatsprüfung, Düsseldorf 1961.

Schwerdtfeger, Gunther, Die öffentlich-rechtliche Klausur. Eine Anleitung zur Lösung öffentlich-rechtlicher Fälle, in: JuS 1969, S. 472-477.

Schwerdtfeger, Gunther/Schwerdtfeger, Angela, Öffentliches Recht in der Fallbearbeitung. Grundfallsystematik, Methodik, Fehlerquellen, 15. Aufl., München 2018.

Seidel, Achim/Reimer, Ekkehart/Möstl, Markus, Allgemeines Verwaltungsrecht, 3. Aufl., München 2019.

Stelkens, Paul/Bonk, Heinz Joachim/Sachs, Michael (Hrsg.), Verwaltungsverfahrensgesetz, 9. Aufl., München 2018.

Stern, Klaus, Verwaltungsprozessuale Grundprobleme in der öffentlichrechtlichen Arbeit, in: JuS 1965, S. 139-145.

Stern, Klaus/Blanke, Hermann-Josef, Verwaltungsprozessrecht in der Klausur, 9. Aufl., München 2008.

Stolleis, Michael, $\$ 2$ Entwicklungsstufen der Verwaltungsrechtswissenschaft, in: Hoffmann-Riem/ Schmidt-Aßmann/Voßkuhle (Hrsg.), Grundlagen des Verwaltungsrechts, Bd. 1, 2. Aufl., München 2012, S. 65-121.

Thieme, Werner/Bull, Hans Peter, Zur Systematik öffentlich-rechtlicher Ansprüche, in: JZ 1969, S. 422426.

Uerpmann-Wittzack, Robert, Examens-Repetitorium Allgemeines Verwaltungsrecht mit Verwaltungsprozessrecht, 5. Aufl., Heidelberg 2018.

Ule, Carl Hermann, Anleitung zum Lösen öffentlichrechtlicher Fälle, in: JuS 1961, S. 190-197.

Vogel, Klaus, Der Verwaltungsrechtsfall. Praktische Einführung in die Bearbeitung verwaltungsrechtlicher Klausuren und Hausarbeiten, Berlin u. a. 1960; 8. Aufl., München 1980.

Vogel, Klaus, Die verwaltungsrechtliche Hausarbeit. Zwei Originalhausarbeiten aus dem Referendarexamen mit einer Einleitung, Berlin u. a. 1965.

Voßkuble, Andreas/Kaiser, Anna-Bettina, Das subjektiv-öffentliche Recht, in: JuS 2009, S. 16-18.

Wahl, Rainer, Herausforderungen und Antworten: Das Öffentliche Recht der letzten fünf Jahrzehnte, Berlin 2006.

Weides, Peter, Verwaltungsverfahren und Widerspruchsverfahren, 3. Aufl., München 1993.

Weimar, Wilhelm, Staats- und Verwaltungsrecht. Lehrfälle mit Lösungen und Grundsatzentscheidungen, Köln 1959.

Winkler, Markus, Klausurtraining Besonderes Verwaltungsrecht, 2. Aufl., Baden-Baden 2017.

Wolf, Joachim, Fälle und Lösungen zum Öffentlichen Recht, Tübingen 2018.

Wolff, Hans J./Bachof, Otto/Stober, Rolf/Kluth, Winfried, Verwaltungsrecht I, 13. Aufl., München 2017.

Würfel, Martin, Das Reichsjustizprüfungsamt, Tübingen 2018. 